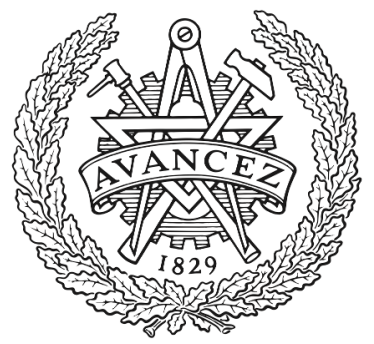

CHALMERS

UNIVERSITY OF TECHNOLOGY

\title{
Smallest scale of wrinkles of a Huygens front in extremely strong turbulence
}

Downloaded from: https://research.chalmers.se, 2023-04-26 14:01 UTC

Citation for the original published paper (version of record):

Sabelnikov, V., Lipatnikov, A., Troshin, A. (2021). Smallest scale of wrinkles of a Huygens front in extremely strong turbulence. PHYSICAL REVIEW E, 104(4).

http://dx.doi.org/10.1103/PhysRevE.104.045101

N.B. When citing this work, cite the original published paper. 


\title{
Smallest scale of wrinkles of a Huygens front in extremely strong turbulence
}

\author{
V. A. Sabelnikov $\odot,{ }^{1,2}$ A. N. Lipatnikov $\odot,{ }^{3}$ and A. I. Troshin $\oplus^{2,4}$ \\ ${ }^{1}$ ONERA - The French Aerospace Laboratory, BP 80100, FR-91123 Palaiseau, France \\ ${ }^{2}$ Central Aerohydrodynamic Institute (TsAGI), 140180 Zhukovsky, Moscow Region, Russian Federation \\ ${ }^{3}$ Department of Mechanics and Maritime Sciences, Chalmers University of Technology, 41296 Gothenburg, Sweden \\ ${ }^{4}$ Moscow Institute of Physics and Technology, 141700 Dolgoprudny, Russia
}

(Received 24 June 2021; revised 8 September 2021; accepted 20 September 2021; published 11 October 2021)

\begin{abstract}
By analyzing the statistically stationary stage of propagation of a Huygens front in homogeneous, isotropic, constant-density turbulence, a length scale $l_{0}$ is introduced to characterize the smallest wrinkles on the front surface in the case of a low constant speed $u_{0}$ of the front when compared to the Kolmogorov velocity $u_{K}$. The length scale is derived following a hypothesis of dynamical similarity that highlights a balance between (i) creation of a front area due to advection and (ii) destruction of the front area due to propagation. Consequently, the front speed is compared with the magnitude of the fluid velocity difference in two points separated by a distance smaller than the Kolmogorov length scale. Appropriateness of the smallest wrinkle scale is demonstrated by applying a fractal approach to evaluating the mean area of the instantaneous front surface. Since the scales of the smallest and larger wrinkles belong to different subranges (dissipation and inertial, respectively) of the Kolmogorov turbulence spectrum, the front is hypothesized to be a bifractal characterized by two different fractal dimensions in the two subranges. Both fractal dimensions are evaluated adapting the aforementioned hypothesis of dynamical similarity. Such a bifractal model yields a linear relation between the mean fluid consumption velocity, which is equal to the front speed $u_{0}$ multiplied with a ratio of the mean area of the instantaneous front surface to the transverse projected area, and the rms turbulent velocity $u^{\prime}$ even if a ratio of $u_{0} / u^{\prime}$ tends to zero.
\end{abstract}

DOI: 10.1103/PhysRevE.104.045101

\section{INTRODUCTION}

The problem of propagation of Huygens front (henceforth, front for brevity) in a flow, described by level sets of a solution to the nonlinear, first-order, Hamilton-Jacobi equation [1,2], is omnipresent in various areas of modern physics. This problem is addressed adopting differently named mathematical tools, e.g., eikonal equation in geometric optics $[3,4]$ and geophysics [5] or $G$ equation [6-8] in combustion science. In particular, the problem of front propagation in randomly advected media [9-12] is straightforwardly relevant, e.g., to autocatalytic reactions in liquids $[13,14]$, and, as a toy problem, to various phenomena, ranging from turbulent combustion [15] and deflagration-to-detonation transition [16] under terrestrial conditions to evolution of thermonuclear Ia supernovae $[17,18]$ in the Universe. Historically, the problem attracted much attention since the 1940s when significant acceleration of premixed flame propagation by turbulence was found. The phenomenon was explained by Damköhler [19] and Shelkin [20], who highlighted random advection of a thin (when compared to the Kolmogorov length scale in contemporary terms) flame by turbulent flow and reduced the influence of the turbulence on the flame to an increase in the area of the flame surface wrinkled due to velocity fluctuations.

By virtue of the nonlinearity of the problem, such a front can develop singularities in finite time, e.g., due to emergence of cusps, isolated pockets with fresh fluid, and annihilation of colliding front elements. Consequently, in the general case, the front is a multivalued (i.e., a ray parallel to the mean direction of the front propagation can cross the front several times), not necessarily connected function, even if the initial front is set using a single-valued function. Therefore, a rigorous theoretical approach to study the evolution of the front surface area in a turbulent flow is strongly limited and a common approach to exploring the front propagation relies on obtaining a global description of the phenomenon. For instance, various models of statistically stationary regime of front propagation in a turbulent flow express the mean velocity $\bar{u}_{T}$ of fluid volume consumption per unit transverse projected area to be a function of the front speed $u_{0}$ and the rms turbulent velocity $u^{\prime}$, with a ratio of $\bar{u}_{T} / u_{0}$ being controlled by an increase in the mean surface area of the instantaneous front $[19,20]$. For instance, such expressions are widely used in the turbulent combustion literature [21]. Moreover, a recent direct numerical simulation study [22] of self-propagation of a passive interface in constant-density turbulence showed a linear relation between the difference $\bar{u}_{T}-u_{0}$ and $u^{\prime}$, at least at $0.5 \leqslant u^{\prime} / u_{0} \leqslant 10$ and moderate turbulent Reynolds numbers.

To analytically determine the mean area of the instantaneous front surface, a spectrum of the front-surface wrinkles is required. A rigorous result for this spectrum has not yet been obtained due to the highly nonlinear and multiscale nature of the wrinkling phenomenon. A common physical approach to evaluating the area is based on assuming self-similarity of the front wrinkles in a wide range of scales and introducing two different length scales that characterize the largest and 
smallest front wrinkles [10,12,23-25]. For instance, Peters [23] and, subsequently, Kerstein [24,25] predicted the linear dependence of $\bar{u}_{T}$ on $u^{\prime}$ by applying a fractal approach to analyzing the case of $u_{0} \ll u^{\prime}$, with $u_{0}$ being on the order of magnitude of velocity fluctuations in the Kolmogorov inertial subrange, i.e., larger than the Kolmogorov velocity $u_{K}=u^{\prime} \operatorname{Re}_{T}^{-1 / 4}$. Here, $\operatorname{Re}_{T}=u^{\prime} L / v$ is the turbulent Reynolds number, $L$ is an integral length scale of turbulence, and $v$ is the kinematic viscosity of the fluid. A fractal [26] is solely characterized by its inner, $\epsilon_{\text {in }}$, and outer, $\epsilon_{\text {out }}$, cutoff scales (i.e., the smallest and largest wrinkles scales, respectively), as well as its dimension $D$. Accordingly, if a front surface is hypothesized to be a fractal, the mean consumption velocity is simply equal to [23-25]

$$
\bar{u}_{T}=u_{0} \frac{A_{f}}{A_{0}}=u_{0}\left(\frac{\epsilon_{\text {out }}}{\epsilon_{\text {in }}}\right)^{D-2},
$$

where $A_{f}=A_{0}\left(\epsilon_{\text {out }} / \epsilon_{\text {in }}\right)^{D-2}$ and $A_{0}$ are the mean area of the front surface and the transverse projected area, respectively.

Therefore, a simple scaling (numerical coefficients are omitted in equations in the rest of the present paper) of

$$
\bar{u}_{T}=u^{\prime}
$$

can easily be obtained [23-25] by setting the fractal dimension $D=7 / 3$ (a value of $D=2.35$ has been measured in turbulent clouds and turbulent jets $[27,28]$ and a value of $D=7 / 3$ was theoretically predicted [24] for fronts propagating in the Kolmogorov turbulence [29-31]) and associating the outer and inner cutoff scales with the integral length scale $L$ and the Gibson length scale [23]

$$
L_{G}=L\left(\frac{u_{0}}{u^{\prime}}\right)^{3}, \eta_{K} \ll L_{G} \ll L,
$$

respectively. The latter scale is obtained from the following constraint:

$$
u_{0}=\left|\Delta u\left(l=L_{G}\right)\right|, u_{K} \ll u_{0} \ll u^{\prime},
$$

where $|\Delta u(l)|$ is the magnitude of velocity difference in two points, separated by the distance $l$. Indeed, for the inertial subrange of Kolmogorov turbulence [29-31],

$$
|\Delta u(l)|=u_{K}\left(\frac{l}{\eta_{K}}\right)^{1 / 3}=u^{\prime}\left(\frac{l}{L}\right)^{1 / 3}
$$

and Eq. (3) results from substitution of Eq. (5) into Eq. (4). Here, $\eta_{K}=L R e_{T}^{-3 / 4}$ is the Kolmogorov length scale.

It is worth noting two points. First, Eq. (4) is a mathematical formulation of the balance of propagation and advection processes at the inner cutoff scale $L_{G}$. These processes are characterized using $u_{0}$ and $\left|\Delta u\left(l=L_{G}\right)\right|$, respectively. Second, under statistically stationary conditions, such a balance should also hold at larger length scales $[32,33]$ by virtue of self-similarity of a fractal structure [26]. For instance, Eq. (1) can be factorized as follows:

$$
\bar{u}_{T}=u_{0}\left(\frac{l}{\epsilon_{\text {in }}}\right)^{D-2}\left(\frac{\epsilon_{\text {out }}}{l}\right)^{D-2}=u_{l}\left(\frac{\epsilon_{\text {out }}}{l}\right)^{D-2},
$$

where $u_{l}=u_{0}\left(l / \epsilon_{\text {in }}\right)^{D-2}$ is associated with the front propagation velocity resulting from the joint action of all eddies whose length scale is less than or equal to $l$. Under conditions of $u_{K} \ll u_{0} \ll u^{\prime}$, this velocity and a hypothesis of dynamical similarity ("the advection and propagation processes balance at all length scales in the fractal range") were used by Kerstein [24] to evaluate the fractal dimension $D$ by comparing characteristic timescales of advection and propagation. For $D=7 / 3$ and $\epsilon_{\text {in }}=L_{G}$, the velocity $u_{l}$ is equal to

$$
u_{l}=u_{0}\left(\frac{l}{L_{G}}\right)^{1 / 3}=u_{0}\left(\frac{l}{L\left(u_{0} / u^{\prime}\right)^{3}}\right)^{1 / 3}=u^{\prime}\left(\frac{l}{L}\right)^{1 / 3}=|\Delta u(l)|,
$$

which is an extension of the balance equation (4) to scales larger than the Gibson scale.

Thus, within the framework of the considered fractal model [23-25], the smallest scale of wrinkles of the front is equal to the Gibson length scale $L_{G}$ provided that $u_{K} \ll u_{0}$. However, the present authors are not aware of an estimate of the smallest scale of wrinkles of the front in the case of very intense turbulence characterized by $u_{K} \gg u_{0}$, i.e., when $u_{0}$ is on the order of velocity fluctuations from the Kolmogorov dissipation range, where the velocity field is smooth. The goals of this paper are (i) to bridge this knowledge gap by estimating the smallest length scale in extremely strong $\left(u_{K} \gg u_{0}\right)$ turbulence, and (ii) to demonstrate appropriateness of the introduced smallest wrinkle scale by applying a bifractal model to the front surface.

\section{SMALLEST LENGTH SCALE FOR A SLOW FRONT}

If $u_{K} \gg u_{0}$, the hypothesis of dynamical similarity and Eq. (4) can still be used to estimate the smallest length scale $l_{0}$ of the front wrinkles. However, Eq. (5) does not hold if $u_{K} \gg u_{0}$. Indeed, in order for $|\Delta u(l)|$ to be equal to $u_{0}$ in so intense turbulence, the distance $l$ should be smaller than the Kolmogorov length scale $\eta_{K}$ and, consequently, $l$ should be associated with the Kolmogorov dissipation subrange. Under such conditions, the velocity field is smooth, and the velocity difference may be estimated as follows [30]:

$$
|\Delta u(l)|=\left|\frac{\partial u}{\partial l}\right| l=\frac{l}{\tau_{K}}=u_{K} \frac{l}{\eta_{K}},
$$

where $\tau_{K}=\eta_{K} / u_{K}$ is the Kolmogorov timescale. At $l \rightarrow$ $\eta_{K}$, both Eq. (5) and Eq. (8) yield the same result, i.e., $\left|\Delta u\left(\eta_{K}\right)\right| \rightarrow u_{K}$, but the latter equation yields the linear dependence of the magnitude $|\Delta u(l)|$ of velocity difference on the scale $l$ in the dissipation subrange $\left(l<\eta_{K}\right)$, whereas dependence of $|\Delta u(l)|$ on $l$ is nonlinear in the inertial subrange $\left(l>\eta_{K}\right)$.

Substitution of Eq. (8) into Eq. (4) results in

$$
\begin{gathered}
u_{0}=\left|\Delta u\left(l_{0}\right)\right|=\frac{l_{0}}{\tau_{K}}=u_{K} \frac{l_{0}}{\eta_{K}}, \\
l_{0}=\eta_{K} \frac{u_{0}}{u_{K}}=u_{0} \tau_{K} \ll \eta_{K} .
\end{gathered}
$$

Equation (10) introduces a length scale of the tiniest wrinkles of front surface under the considered conditions of $u_{K} \gg$ $u_{0}$ and $\operatorname{Re}_{T} \gg 1$. 


\section{FRONT AS A BIFRACTAL}

To show a physics brought by the introduced smallest wrinkle scale $l_{0}$, let us consider the influence of a decrease in a ratio of $u_{0} / u^{\prime}$ on the fractal characteristics of the front. When $u_{0}$ becomes smaller than $u_{K}$ and much smaller than $u^{\prime}$, the front is wrinkled not only by turbulent eddies from the inertial subrange $\left(\eta_{K}<l<L\right)$, but also by eddies from the dissipation subrange $\left(l_{0}<l<\eta_{K}\right)$. If the front is assumed to be a fractal in each of this subrange, the fractal dimensions should be different in the two subranges characterized by different turbulence spectra [30,31]. Accordingly, when the ratio of $u_{0} / u^{\prime}$ is decreased, transition from a monofractal to a bifractal front surface should occur at certain value of $u_{0} / u^{\prime}$, associated with the unity order of $u_{0} / u_{K}$. At lower values of $u_{0} / u^{\prime}$, the front is a bifractal, similarly to scalar isoconcentration surfaces in the vicinity of turbulent-nonturbulent interfaces in the case of a large Schmidt number. The latter problem was explored by Sreenivasan et al. [34], who argued that the fractal dimension of scalar isosurfaces should be different for scales larger and smaller than the Kolmogorov length scale; see Figs. 2(a) and 6 in the cited paper.

For a front hypothesized to be a bifractal, (i) the inner cutoff scale is equal to $l_{0}$, (ii) a crossover scale (the outer cutoff for the small-scale fractal and the inner cutoff for the larger-scale fractal) is equal to $\eta_{K}$, and (iii) the outer cutoff scale is equal to $L$. Consequently, the area ratio and the mean consumption velocity are equal to

$$
\begin{gathered}
\frac{A_{f}}{A_{0}}=\left(\frac{\eta_{K}}{l_{0}}\right)^{D_{s}-2}\left(\frac{L}{\eta_{K}}\right)^{D_{l}-2}, \\
\bar{u}_{T}=u_{0} \frac{A_{f}}{A_{0}}=u_{0}\left(\frac{\eta_{K}}{l_{0}}\right)^{D_{s}-2}\left(\frac{L}{\eta_{K}}\right)^{D_{l}-2},
\end{gathered}
$$

respectively. Here, the fractal dimensions $D_{l}$ and $D_{s}$ characterize the large-scale $\left(\eta_{K}<l<L\right)$ and small-scale $\left(l_{0}<l<\right.$ $\eta_{K}$ ) subranges, respectively.

For the front studied here, the large-scale subrange fractal dimension could be set following common practice, i.e., $D_{l}=7 / 3$ [23-28]. As shown by Kerstein [24] and discussed briefly in Sec. I, this fractal dimension is linked with the dynamical similarity in the inertial subrange of Kolmogorov turbulence. The small-scale subrange fractal dimension $D_{s}$ could be evaluated by adapting the same hypothesis to the dissipation subrange $\left(l<\eta_{K}\right)$. Within the framework of the bifractal model, small-scale eddies characterized by a length scale equal to or smaller than $l$ result in increasing the front propagation velocity from $u_{0}$ to $u_{0}\left(l / l_{0}\right)^{D_{s}-2}$. The magnitude $|\Delta u(l)|$ of velocity difference for the small-scale subrange is estimated with Eq. (8). By equating $u_{0}\left(l / l_{0}\right)^{D_{s}-2}$ and $|\Delta u(l)|$, we arrive at

$$
u_{0}\left(\frac{l}{l_{0}}\right)^{D_{s}-2}=\frac{l}{\tau_{K}} .
$$

Equation (13) holds solely if $D_{s}=3$. Therefore, the smallscale wrinkles of the front surface are packed so densely that they fill the volume of a spatial layer of a thickness $\eta_{K}\left(\eta_{K} \gg l_{0}\right)$ adjacent to the front surface measured with resolution $\eta_{K}$. Attention of the first author to this issue was attracted by Professor E. Hawkes (The University of New
South Wales, Sydney, Australia) during a discussion at the 8th European Combustion Meeting in Dubrovnik, Croatia on April 17th, 2017. Space-filling surfaces characterized by $D=$ 3 are discussed, e.g., by Ahmed and Bokhari [35]. The same limiting value of $D_{s}=3$ was also proposed by Sreenivasan and Prasad [36] to satisfy Schmidt number similarity requirement at $S c \rightarrow \infty$ within the framework of the aforementioned bifractal model for scalar isosurfaces [34].

Substitution of $D_{l}=7 / 3$ and $D_{s}=3$ into Eq. (12) yields

$$
\begin{aligned}
\bar{u}_{T} & =u_{0} \frac{\eta_{K}}{l_{0}}\left(\frac{L}{\eta_{K}}\right)^{1 / 3}=u_{K}\left(\frac{L}{\eta_{K}}\right)^{1 / 3}=u^{\prime} \operatorname{Re}_{T}^{-1 / 4}\left(\operatorname{Re}_{T}^{3 / 4}\right)^{1 / 3} \\
& =u^{\prime}
\end{aligned}
$$

Thus, in the considered case at $u_{0} \rightarrow 0$, the mean consumption velocity is still proportional to $u^{\prime}$. The same asymptotic $\left(u_{0} \ll u^{\prime}\right)$ scaling of $\bar{u}_{T}=u^{\prime}$ was recently published by Mayo and Kerstein [12], who addressed the case of a single-scale random velocity field, whereas a multiscale Kolmogorov velocity field is studied in the present work.

It is worth noting that the fractal dimensions $D_{l}$ and $D_{s}$ can be evaluated using Eq. (6), where $\epsilon_{\text {out }}=L, D=D_{l}$, and

$$
\begin{aligned}
u_{l} & =u_{0}\left(\frac{\eta_{K}}{l_{0}}\right)^{D_{s}-2}\left(\frac{l}{\eta_{K}}\right)^{D_{l}-2} \\
& =u_{0}\left(\frac{u_{K}}{u_{0}}\right)^{D_{s}-2}\left(\frac{l}{L}\right)^{D_{l}-2}\left(\frac{L}{\eta_{K}}\right)^{D_{l}-2} \\
& =u_{0}\left(\frac{u^{\prime}}{u_{0}} \operatorname{Re}_{T}^{-1 / 4}\right)^{D_{s}-2}\left(\frac{l}{L}\right)^{D_{l}-2}\left(\operatorname{Re}_{T}^{3 / 4}\right)^{D_{l}-2} \\
& =u_{0}\left(\frac{u^{\prime}}{u_{0}}\right)^{D_{s}-2} \operatorname{Re}_{T}^{-D_{s} / 4+1 / 2+3 D_{l} / 4-3 / 2}\left(\frac{l}{L}\right)^{D_{l}-2}
\end{aligned}
$$

in the inertial subrange $\left(\eta_{K}<l<L\right)$. In order for front propagation and advection to balance one another at the scale $l$, the "subfilter" propagation velocity $u_{l}$ should be equal to the magnitude $|\Delta u(l)|$ of velocity difference for the inertial subrange; see Eq. (5). Consequently,

$$
u_{0}\left(\frac{u^{\prime}}{u_{0}}\right)^{D_{s}-2} \operatorname{Re}_{T}^{-D_{s} / 4+1 / 2+3 D_{l} / 4-3 / 2}\left(\frac{l}{L}\right)^{D_{l}-2}=u^{\prime}\left(\frac{l}{L}\right)^{1 / 3} \text {. }
$$

This equality holds for various $u^{\prime} / u_{0}, R e_{T}$, and $l / L$ only if $D_{s}=3$ and $D_{l}=7 / 3$. In this case, $-D_{s} / 4+3 D_{l} / 4-1=0$ and the $R e_{T}$ term vanishes in Eq. (16).

Note that the second equality in Eq. (14), i.e., $\bar{u}_{T}=$ $u_{K}\left(L / \eta_{K}\right)^{1 / 3}$, looks like an expression for the mean consumption velocity yielded by a single-fractal model for a front whose self-propagation velocity is equal to $u_{K}$. In other words, the small-scale fractal of the considered bifractal manifests itself in an increase in the front velocity from $u_{0}$ to $u_{K}$. This result is consonant with an earlier hypothesis [37] that front propagation velocity becomes equal to the Kolmogorov velocity in very intense turbulence.

\section{CONCLUDING REMARKS}

The statistically stationary stage of propagation of Huygens front in homogeneous, isotropic, constant-density 
turbulence is explored in the case of a low constant speed $u_{0}$ of the front when compared to the Kolmogorov velocity $u_{K}$. A length scale $l_{0}<\eta_{K}$ of the smallest wrinkles on the front surface is introduced by highlighting a balance of advection and propagation processes and, consequently, comparing the front speed with the magnitude of the fluid velocity difference in two points separated by a distance smaller than the Kolmogorov length scale.

To evaluate the area of the front surface, a fractal approach is applied. Since the scales of the smallest $\left(l_{0}<l<\eta_{K}\right)$ and larger $\left(\eta_{K}<l<L\right)$ wrinkles belong to different subranges (dissipation and inertial, respectively) of the Kolmogorov turbulence spectrum, the two types of wrinkles should have different fractal dimensions. Accordingly, transition from a monofractal front surface with fractal dimension $D=7 / 3$ at $u_{0} / u^{\prime}>\operatorname{Re}_{T}^{-1 / 4}$ to a bifractal with two different fractal dimensions occurs with decreasing a ratio of $u_{0} / u^{\prime}$, i.e., at $u_{0} / u^{\prime}<\operatorname{Re}_{T}^{-1 / 4}$. The two fractal dimensions of the bifractal are evaluated by adapting a hypothesis of dynamical similarity, i.e., by requiring a balance of the local (in the spectral sense) changes of the front area due to its propagation and advection by the flow. Such a bifractal model yields a linear relation [see Eq. (14)] between the mean velocity $\bar{u}_{T}$ of fluid consumption and the rms turbulent velocity $u^{\prime}$ even if a ratio of $u_{0} / u^{\prime}$ tends to zero.

It should be stressed that the present analysis not only yields a simple scaling of $\bar{u}_{T}=u^{\prime}$ at the limit of $u_{0} / u^{\prime} \rightarrow 0$ in the Kolmogorov turbulence, but additionally suggests a potential importance of the introduced length scale of the smallest front wrinkles in the rigorous theoretical approach to studying the growth of the front surface area. The introduced length scale could directly be relevant to modeling autocatalytic reactions in liquids characterized by a very large Schmidt number. If a front thickness is comparable with or larger than the Kolmogorov length scale due to molecular transport processes, the smallest front wrinkles are expected to be controlled by molecular mixing. Nevertheless, even in such a case, a slowly propagating front can be bifractal [38] if $R e_{T} \gg 1$ and a timescale characterizing processes in the front, including molecular transport, is much smaller than the integral timescale of the turbulence (the Damköhler number $D a \gg 1)$ and is much larger than the Kolmogorov timescale (the Karlovitz number $K a \gg 1$ ). Moreover, under such conditions, the mean front speed scales as $u^{\prime}$, as discussed in detail elsewhere [38].

\section{ACKNOWLEDGMENTS}

V.A.S. was financially supported by ONERA and by the Grant of the Ministry of Education and Science of the Russian Federation (Contract No. 14.G39.31.0001). A.N.L. was supported by the Chalmers Combustion Engine Research Center (CERC). A.I.T. was financially supported by the Grant of the Ministry of Education and Science of the Russian Federation (Contract No. 14.G39.31.0001). V.A.S. is grateful to E. Hawkes for valuable discussions. We are grateful to the reviewers for valuable comments, which helped us to substantially improve the paper.
[1] J. A. Sethian, Level Set Methods and Fast Marching Methods: Evolving Interfaces in Computational Geometry, Fluid Mechanics, Computer Vision, and Materials Science (Cambridge University Press, Cambridge, UK, 1999).

[2] S. J. Osher and R. Fedkiw, Level Set Methods and Dynamic Implicit Surfaces (Springer, Heidelberg, 2003).

[3] D. T. Paris and F. K. Hurd, Basic Electromagnetic Theory (McGraw-Hill, New York, 1969).

[4] M. Born and E. Wolf, Principles of Optics: Electromagnetic Theory of Propagation, Interference, and Diffraction of Light, 7th ed. (Cambridge University Press, Cambridge, UK, 1999).

[5] C. J. Ammon, A. A. Velasco, T. Lay, and T. C. Wallace, Foundations of Modern Global Seismology, 2nd ed. (Academic Press, London, 2020).

[6] L. D. Landau, Acta Physicochim. USSR 19, 77 (1944).

[7] G. H. Markstein, Nonsteady Flame Propagation (Pergamon Press, Oxford, UK, 1964).

[8] A. R. Kerstein, Wm. T. Ashurst, and F. A. Williams, Phys. Rev. A 37, 2728 (1988).

[9] A. R. Kerstein and W. T. Ashurst, Phys. Rev. Lett. 68, 934 (1992).

[10] M. Chertkov and V. Yakhot, Phys. Rev. Lett. 80, 2837 (1998).

[11] J. R. Mayo and A. R. Kerstein, Phys. Rev. E 78, 056307 (2008).

[12] J. R. Mayo and A. R. Kerstein, J. Stat. Phys. 176, 456 (2019).

[13] S. S. Shy, P. D. Ronney, S. G. Buckley, and V. Yakhot, Proc. Combust. Inst. 24, 543 (1992).
[14] P. D. Ronney, B. D. Haslam, and N. O. Rhys, Phys. Rev. Lett. 74, 3804 (1995).

[15] S. Chaudhuri, F. Wu, D. Zhu, and C. K. Law, Phys. Rev. Lett. 108, 044503 (2012).

[16] A. Y. Poludnenko, T. A. Gardiner, and E. S. Oran, Phys. Rev. Lett. 107, 054501 (2011).

[17] V. N. Gamezo, A. M. Khokhlov, and E. S. Oran, Phys. Rev. Lett. 92, 211102 (2004).

[18] V. N. Gamezo, A. M. Khokhlov, E. S. Oran, A. Y. Chtchelkanova, and R. O. Rosenberg, Science 299, 77 (2003).

[19] G. Z. Damköhler, Z. Electrochem. Angew. Phys. Chem. 46, 601 (1940).

[20] K. I. Shelkin, NACA Tech. Memo 1110 (1947).

[21] T. Poinsot and D. Veynante, Theoretical and Numerical Combustion, 2nd ed. (Edwards, Philadelphia, 2005).

[22] R. Yu, X.-S. Bai, and A. N. Lipatnikov, J. Fluid Mech. 772, 127 (2015).

[23] N. Peters, Proc. Combust. Inst. 21, 1231 (1986).

[24] A. R. Kerstein, Combust. Sci. Technol. 60, 441 (1988).

[25] A. R. Kerstein, Phys. Rev. A 44, 3633 (1991).

[26] B. B. Mandelbrot, Fractals (W.H. Freeman, San Francisco, CA, 1977).

[27] H. G. E. Hentschel and I. Procaccia, Phys. Rev. A 29, 1461 (1984).

[28] K. Sreenivasan, R. Prasad, C. Meneveau, and R. Ramshankar, Pure Appl. Geophys. 131, 43 (1989).

[29] A. N. Kolmogorov, Dokl. Akad. Nauk SSSR 30, 299 (1941). 
[30] A. S. Monin and A. M. Yaglom, Statistical Fluid Mechanics: Mechanics of Turbulence 2 (The MIT Press, Cambridge, MA, 1975).

[31] U. Frisch, Turbulence. The Legacy of A.N. Kolmogorov (Cambridge University Press, Cambridge, UK, 1995).

[32] A. Y. Klimenko, Combust. Sci. Technol. 139, 15 (1998).

[33] A. Y. Klimenko, Phys. Fluids 33, 025112 (2021).
[34] K. R. Sreenivasan, R. Ramshankar, and C. Meneveau, Proc. R. Soc. London A 421, 79 (1989).

[35] M. Ahmed and S. Bokhari, IEEE Trans. Parallel Distrib. Syst. 18, 1258 (2007).

[36] K. R. Sreenivasan and R. R. Prasad, Physica D 38, 322 (1989).

[37] J. Chomiak, Prog. Energy Combust. Sci. 5, 207 (1979).

[38] V. A. Sabelnikov and A. N. Lipatnikov, Phys. Fluids 32, 095118 (2020). 